\title{
Tofacitinib y la necesidad de vigilancia de potenciales efectos adversos en pacientes con artritis reumatoide
}

Sixto Zegarra Mondragón*

Servicio de Reumatología, Hospital Universitario Ramón y Cajal, Madrid, España.

*Correspondencia: hazdehis_175@hotmail.com

\section{Resumen}

Tofacitinib es un inhibidor de JAK-quinasa indicado en el tratamiento de la artritis reumatoide (AR), pudiendo administrarse en monoterapia cuando se ha producido fallo o intolerancia a FAMEs y/o anti-TNF. Por otro lado se debe tener en cuenta un balance entre beneficios y riesgos que podría presentar, especialmente el riesgo de infecciones como aparición de herpes zóster, riesgo de activación de VHB latente y de candidiasis, la relación con neoplasias y linfomas; así como el posible rebrote de actividad articular tras su suspensión una vez alcanzada la remisión; motivos por los que exponemos en este texto dichos aspectos concernientes a la utilización de esta nueva diana terapéutica en AR.

Palabras clave:

Tofacitinib, artritis reumatoide.

\section{Introducción}

Tofacitinib es un inhibidor potente y selectivo de la familia de las JAK-quinasas. En las células humanas, tofacitinib inhibe preferentemente las señales de transducción activadas por receptores de citoquinas heterodiméricos que se unen a JAK1 y/o JAK3, con una selectividad funcional superior a la de los receptores de citoquinas que activan señales de transducción a través de pares de JAK2. La inhibición de JAK1 y JAK3 por tofacitinib atenúa las señales de transducción activadas por las interleucinas (IL-2, 4, 6, 7, 9, 15 y 21) y los interferones de tipo I y II, lo que da lugar a la modulación de la respuesta inmune e inflamatoria. [1]

Tofacitinib en dosis de $5 \mathrm{mg}$ dos veces al día está indicado para el tratamiento de la AR activa de moderada a grave, en adultos que han respondido inadecuadamente o que son intolerantes a uno o más FAMEs. Varios ensayos clínicos con un punto de corte para análisis a los 24 meses, mostraron que la monoterapia con tofacitinib (como tratamiento de primera o segunda línea) y la terapia de combinación con un FAME sintético convencional fue eficaz para reducir los signos y síntomas de la enfermedad y mejorar las escalas de calidad de vida relacionada (HAQ) con beneficios sostenidos durante la terapia a largo plazo en un seguimiento que abarcaba un periodo máximo de 96 meses. [2] 
De forma general y según el análisis de los ensayos pivotales de los inhibidores de JAK-quinasas, existe una mayor incidencia de reacciones adversas con la combinación de tofacitinib y metotrexato frente a tofacitinib en monoterapia, debiendo considerarse los riesgos y beneficios antes de comenzar el tratamiento tanto como en los casos de infecciones recurrentes y antecedentes de infecciones graves o de infecciones oportunistas, o en el caso de comorbilidades subyacentes que puedan predisponer a estas infecciones.

Por este motivo, describiremos a manera de resumen, algunos ensayos clínicos y metanálisis que revelan potenciales complicaciones que podrían surgir tras el inicio del tratamiento con tofacitinib en pacientes con AR, desarrollados durante la fase clínica de investigación del fármaco, o reseñados en reportes de la literatura científica.

\section{Tofacitinib y riesgo de reactivación de herpes \\ zóster}

Los pacientes con AR tienen un mayor riesgo de presentar infección por herpes zoster ( $\mathrm{HZ}$ ) y el riesgo parece aumentar en los pacientes tratados con tofacitinib.

Un reporte que consideró a todos los pacientes con AR que recibieron tofacitinib en sus respectivos ensayos clínicos (6192 pacientes, 16839 pacientes-año), informó la aparición de HZ en 636 de ellos (OR: 4; IC 95\%: 3,7- 4,4). En la mayoría de los casos (93\%), HZ se clasificó como no seronegativo, y la mayoría de los pacientes (94\%) tenían afectación únicamente monometamérica. Los odds ratio de $\mathrm{HZ}$ variaron entre las regiones geográficas, con cifras que iban desde 2,4 (IC 95\%: 2-2,9) en Europa del Este hasta 8,0 (IC del 95\%: 6,6-9,6) en Japón y 8,4 (IC del 95\%: 6,4-10,9) en Corea. Además se señalaba que la edad mayor de 65 años, el uso de glucocorticoides y la dosis de tofacitinib fueron factores de riesgo independientes para la aparición de HZ. [3]

En relación con la protección que conferiría la vacunación específica contra el virus de la varicela zoster (VVZ), un ensayo clínico en fase II controlado con placebo que incluyó a 112 pacientes mayores de 50 años con AR activa y que recibían metotrexato de terapia de base, se administró la vacunación a todos los casos incluidos, aleatorizándose un grupo que recibió tofacitinib y otro placebo; llegándose a determinar a las 6 semanas los niveles de IgG específicos de VVZ, sin encontrar diferencias estadísticamente significativas de los niveles de anticuerpos entre ambos grupos y manteniendo respuestas inmunomediadas humorales y celulares similares. [4]

\section{Riesgo de reactivación del virus VHB en pacientes tratados con tofacitinib}

Se ha llegado a describir en un estudio observacional retrospectivo el riesgo de aparición de replicación activa del VHB en pacientes con $A R$ y portadores del antígeno de superficie (HBs-Ag) con niveles bajos de DNA del virus, en quienes no se administró un tratamiento antiviral preventivo con entecavir antes del inicio del tratamiento con tofacitinib. [5]

En contraste, ningún paciente con AR que recibió terapia profiláctica antiviral desarrolló reactivación del VHB, lo que indica la eficacia de la profilaxis antiviral para prevenir la reactivación del virus. De esta forma, se describe la importancia de la vigilancia del riesgo de reactivación del VHB en pacientes con $A R$ que van a iniciar tratamiento con inhibidores de las JAK-quinasas.

\section{Riesgo infecciones fúngicas con tofacitinib}

En el marco del riesgo de infecciones fúngicas, un estudio in vitro ha demostrado el proceso relacionado con la capacidad de tofacitinib para revertir la actividad fungicida potenciada por TNF-alfa e IFN-gamma en células polimorfas murinas, y también la capacidad para suprimir la expresión de la quimiocina CXCL5 y la infiltración de PMN en los tejidos infectados de ratones.

Más importante aún, tofacitinib antagonizó significativamente la capacidad de TNFa, IFN y y GM-CSF para estimular a células polimorfonucleares humanas involucradas en la fagocitosis directa de Candida albicans en cultivos in vitro. [6]

De igual forma, en sus respectivos ensayos clínicos, se ha demostrado aumento de riesgo de infecciones candidiásicas en pacientes con espondiloartropatías y artropatía psoriásica tratados con inhibidores de la IL-17 como secukinumab, por inhibición de mecanismos de respuesta inmune por vías similares.

\section{Neoplasias malignas y tofacitinib}

En un metanálisis de 113 trabajos correspondientes al periodo 2000-2015, el riesgo de aparición de neoplasias malignas en pacientes con AR tratados con agentes biológicos, mostró odds ratios para los anti-TNF: infliximab (1,68; IC 95\%: 0,485,92), etanercept $(0,79$; IC 95\%: 0,44-1,40), adalimumab ( 0,93; IC 95\%: 0,43-2,03), certolizumab (0,87; IC 95\%: 0,282,75), golimumab (0.87; IC 95\%: 0,39-1,95); y en el caso de los no anti-TNF: abatacept (1,04; IC 95\%: 0,32-3,32); rituximab (0,58; IC 95\%: 0,21-1,56), tocilizumab (0,60; IC 95\%: $0,16-2,28)$ y finalmente para tofacitinib (1,15; IC 95\%: 0,24$5,47)$. [7] Además se señala en este metanálisis que existieron diferencias marginales en la tasa de incidencia de tumores 
malignos de órgano sólido y neoplasias hematológicas frente a las neoplasias de piel tipo no melanoma, a diferencia de estudios previos que inclinaban el riesgo de este último tipo de neoplasias en los que recibieron anti-TNF.

\section{Linfoma y tofacitinib}

En el programa de desarrollo clínico de tofacitinib en los pacientes con AR, las tasas de linfoma se mantuvieron estables con el tiempo y hubo diferencias mínimas en las características iniciales de los pacientes con diagnóstico posterior de linfoma frente a los que no lo desarrollaron.

Para este descripción, en un metanálisis realizado en 6194 pacientes que recibieron tofacitinib (19 406 pacientes-año) con una duración media del tratamiento de 3,4 años, se reportaron 19 casos de linfomas, registrando un OR: 0,10 (IC 95\%: 0,06-0,15) sin reportarse un aumento de los pacientes con este diagnóstico en función del tiempo de exposición al fármaco. [8]

En el caso de estos pacientes con $A R$, que recibieron tofacitinib y desarrollaron linfoma, la mayoría tenían síndrome de Sjögren secundario asociado, siendo seropositivos y ACPA (+) al inicio del estudio, frente a los controles emparejados.

\section{Tofacitinib y su suspensión en remisión de AR}

Finalmente, respecto a la posibilidad de descontinuación de tofacitinib después de lograr la remisión o baja actividad en pacientes con $A R$, un estudio que incluyó a 64 pacientes, de los cuales 54 suspendieron el tofacitinib y 10 continuaron el tratamiento con este fármaco, se determinó al final de la semana 52 del seguimiento, que aproximadamente la tercera parte de los pacientes que descontinuaron el tratamiento, se mantuvieron en remisión y/o baja actividad; siendo los niveles bajos de factor reumatoide un elemento predictor para mantenerse sin reactivación de la enfermedad en los pacientes que suspendieron el tratamiento con este inhibidor de la JAK-quinasa. [9]

\section{Conclusión}

De esta forma, queda pendiente aún la inserción en práctica clínica habitual del uso de este fármaco, sea en primera línea o tras fallo a otros tratamientos biológicos, para determinar de forma directa y en seguimiento de cohortes a mayor escala los beneficios terapéuticos y el espectro de potenciales efectos adversos de esta nueva diana en el manejo de la AR.

\section{Referencias}

1. Dhillon S. Tofacitinib: A review in rheumatoid arthritis. Drugs. 2017; 77 (18): 1987-2001.

2. Fleischmann R. Tofacitinib in the treatment of active rheumatoid arthritis in adults. Immunotherapy. 2018; 10(1): 39-56.

3. Winthrop KL, Curtis JR, Lindsey S, Tanaka Y, Yamaoka K, Valdez $\mathrm{H}$, et al. Herpes zoster and tofacitinib: Clinical outcomes and the risk of Concomitant therapy. Arthritis Rheumatol. 2017; 69(10): 1960-8.

4. Winthrop $\mathrm{KL}$, Wouters $A G$, Choy $E H$, et al. The safety and immunogenicity of live zoster vaccination in patients with rheumatoid arthritis before starting tofacitinib: A randomized phase II trial. Arthritis Rheumatol. 2017; 69(10): 1969 -77.

5. Chen YM, Huang WN, Wu YD, et al. Reactivation of hepatitis $B$ virus infection in rheumatoid arthritis patients receiving tofacitinib: a real-world study. Ann Rheum Dis. 2017; 2: 113-22.

6. Chen Y, Gong FY, Li ZJ, et al. A study on the risk of fungal infection with tofacitinib (CP-690550), a novel oral agent for rheumatoid arthritis. Sci Rep. 2017; 7(1): 6779.

7. Maneiro JR, Souto A, Gomez-Reino JJ, et al. Risks of malignancies related to tofacitinib and biological drugs in rheumatoid arthritis: Systematic review, meta-analysis, and network meta-analysis. Semin Arthritis Rheum. 2017; 47(2): 149-56.

8. Mariette X, Chen C, Biswas P, Kwok K, Boy MG, et al. Lymphoma in the tofacitinib rheumatoid arthritis clinical development program. Arthritis Care Res (Hoboken). 2017; Sep 21. doi: 10.1002/acr.23421.

9. Kubo S, Yamaoka K, Amano K, et al. Discontinuation of tofacitinib after achieving low disease activity in patients with rheumatoid arthritis: a multicentre, observational study. Rheumatology (Oxford). 2017; 56(8): 1293-1301.

\section{Publique en Acta Reumatológica}

Acta Reumatológica es una revista que tiene por fin la difusión de estudios clínicos relacionados con aspectos prácticos del diagnóstico, tratamiento y seguimiento de pacientes con patología reumatológica, de estudios epidemiológicos relacionados con patología inflamatoria y musculoesquelética de presentación común o infrecuente en la práctica clínica tanto en población adulta como pediátrica, de casos clínicos de patología poco habitual o de presentaciones inhabituales de patología frecuente, de imágenes didácticas e ilustrativas en reumatología y del estado actual e innovación en la formación especializada en reumatología. 